\title{
Leptogenesis via preheating *
}

\author{
Marco Peloso ${ }^{\text {a }}$

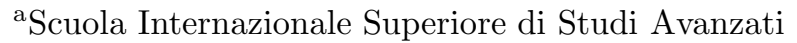 \\ Via Beirut 4, 34014 Trieste, Italy \\ and \\ Istituto Nazionale di Fisica Nucleare, Sez. di Trieste \\ Via Valerio 2, I-34127 Trieste, Italy
}

\begin{abstract}
Leptogenesis constitues a very simple scenario to achieve the baryon asymmetry that we observe today. It requires only the presence of right handed neutrinos (which arise very naturally in many extensions of the Standard Model) and depends crucially on the mechanism responsible for their production. In particular, when their mass exceeds the inflaton mass $\left(10^{13} \mathrm{GeV}\right.$ in chaotic inflation) only non perturbative production can occur. It is shown here that non perturbative production of fermions in an expanding Universe is a very efficient mechanism up to masses of order of $\left(10^{17}-10^{18}\right) \mathrm{GeV}$, and that it can be easily applied to solve the baryon asymmetry problem.
\end{abstract}

\section{Introduction}

Inflation is at present the most natural and accepted solution to many cosmological puzzles, such as the flatness, the horizon, and the monopole problems. However, the Universe after inflation looks very different from the one we observe today, since the huge expansion leaves it empty of radiation and matter.

The only form of energy which is not diluted away after this phase is the vacuum energy of the field (or fields) which leads inflation itself, namely the inflaton field. The process of converting this energy into the radiation and matter which we see around has been named reheating and it has been the object of intense studies in the past decades.

Apart from this energy conversion, the processes which govern the first instant of the Universe must also favor the production of matter over antimatter. Indeed, on one hand direct observations put strong limits to the presence of antimatter in our cluster, while on the other considerations about how the light elements abundances were formed when the Universe was about $1 \mathrm{MeV}$ hot lead us to conclude that the difference between the number density of baryons and that of antibaryons is about $10^{-10}$ if normalized to the entropy density of the Universe.

Until now, many mechanisms for the generation of the baryon $(B)$ asymmetry have been proposed (for a recent review, see [2]).

Grand Unified Theories (GUTs) unify the strong and the electroweak interactions and predict baryon number violation at the tree level. They are - therefore - perfect candidates for a theory of baryogenesis. There, the out-ofequilibrium decay of superheavy particles can explain the observed baryon asymmetry.

Another plausible scenario is the theory of electroweak baryogenesis (in the context of the Minimal Supersymmetric Standard Model), where baryon number violations take place at the quantum level due to the usoppressed and baryon number violating sphalerons in the hot plasma.

Sphaleron transitions leave unchanged the combination $B-L$ (where $L$ is the lepton number) and play the role of converting an initial lepton asymmetry to a final mixture of nonvanishing $B$ and $L$. This is the key idea of the theories of baryogenesis via leptogenesis. Once a lepton asymmetry is produced, sphaleronic transitions convert a fraction of it into baryon number. In the Standard Model the following relation for the

\footnotetext{
*Talk given at Valencia '99, based on a paper by G.F.
} Giudice, M. Peloso, A. Riotto and I. Tkachev 11. 
final amount of the baryon asymmetry holds:

$B=\left(\frac{8 n_{f}+4 n_{H}}{22 n_{f}+13 n_{H}}\right)(B-L)$,

where $n_{H}$ is the number of Higgs doublets and $n_{f}$ is the number of fermion generations.

Adding right-handed Majorana neutrinos to the SM breaks $B-L$, and the primordial lepton asymmetry may be generated by their outof-equilibrium decay. This simple extension of the SM can be embedded into GUTs with gauge groups containing $S O(10)$. Heavy right-handed Majorana neutrinos are particularly welcome to explain the smallness of the light neutrino masses via the see-saw mechanism [3]. In most of the models proposed so far to generate neutrino masses, the latter are of the Majorana type, which implies the existence of interactions which violate the lepton number and thus render leptogenesis particularly attractive.

The leptogenesis scenario depends crucially on the details of the reheating process after inflation and on the production mechanism that was responsible for populating the Universe with righthanded neutrinos.

The simplest way to envision this reheating process is if the comoving energy density in the zero mode of the inflaton decays perturbatively into ordinary particles, which then scatter and thermalize to form a thermal background [4.5]. Of particular interest is a quantity known as the reheat temperature, which is given by

$T_{R H}=\sqrt{\Gamma_{\phi} M_{p}}$,

where $\Gamma_{\phi}$ is the inflaton decay rate.

In order to produce a sufficient amount of heavy right-handed neutrinos with mass $M_{N}$, it is commonly assumed that $T_{R H} \gtrsim M_{N}$.

However, $T_{R H}$ is constrained by the so called gravitino problem [6] to be less than about $10^{10} \mathrm{GeV}$.

This bound increases if the heavy neutrinos are produced directly through the inflaton decay process. This would be kinematically accessible only if 7

$M_{N}<M_{\phi} \simeq 10^{13} \mathrm{GeV}$.

${ }^{2}$ The last equality is required by the observed density and temperature fluctuations.
The outlook for leptogenesis might be brightened even further with the realization that reheating may differ significantly from the simple picture described above [7,8]. In the first stage of reheating (namely in the first dozen or so oscillations), called preheating [7], nonlinear quantum effects may lead to extremely effective dissipative dynamics and explosive particle production, even when single particle decay is kinematically forbidden.

It has been already shown that preheating plays an extremely important role in baryogenesis, in particular for the production of superheavy GUT bosons [9 [10]. The application of preheating that will be instead presented here is a very new one [1] and it consists in enriching the leptogenesis scenario with the possibility of producing right-handed neutrinos which are too heavy to be generated in the usual perturbative ways.

In what follows it will be shown (both with numerical calculations and with some analytical estimates 3 that the production of a generic superheavy fermionic field during preheating is a very efficient mechanism up to masses of order of $10^{18} \mathrm{GeV}$. As a consequence, it will be pointed out that a sufficient $L$ asymmetry can be generated if the fermionic field is a right-handed neutrino.

\section{Production of fermions during preheat- ing}

The coupling inflaton - fermions gives to the latter the effective time dependent mass

$m(t)=m_{X}+g \phi(t)$,

where $m_{X}$ is the bare fermionic mass.

This changing mass induces the creation of $X$ particles. To see this, one has to diagonalize the hamiltonian associated to the Dirac equation

$$
\left(\frac{i}{a} \gamma^{\mu} \partial_{\mu}+i \frac{3}{2} H \gamma^{0}-m\right) X=0
$$

that is written here for an expanding flat Universe ( $H$ is the Hubble constant) and in conformal time $\eta$.

\footnotetext{
${ }^{3}$ New analytical expressions with respect to the paper 1 - where the reader is addressed for a more general treatment of leptogenesis — will be presented here.
} 
Once the diagonalization is performed (see [1] for details) the number of produced fermions is given by

$$
\begin{aligned}
n(\eta) & =\frac{1}{\pi^{2} a^{3}(\eta)} \int_{0}^{\infty} d k k^{2} n_{k}^{2}(\eta) \\
n_{k}^{2}(\eta) & =\frac{\omega-2 k \operatorname{Re}\left(u_{+}^{*} u_{-}\right)-a m\left(1-2 u_{+}^{*} u_{+}\right)}{2 \omega}(6)
\end{aligned}
$$

where $\omega^{2}=k^{2}+m(t)^{2} a^{2}, k$ is the momentum of the produced particle, and $a$ is the scale factor of the Universe. The functions $u_{ \pm}$are, respectively, the positive and negative eigenfunctions of $\gamma_{0}$ which enter in the decomposition of the fermionic field. They satisfy the uncoupled equations

$$
\left[\frac{d^{2}}{d \eta^{2}}+\omega^{2} \pm i\left(a^{\prime} m+a m^{\prime}\right)\right] u_{ \pm}(k)=0,
$$

with initial conditions at time $\eta=0$ given by

$$
\begin{aligned}
& u_{ \pm}(0)=\sqrt{\frac{1}{2}\left(1 \mp \frac{m a}{\omega}\right)} \\
& u_{ \pm}^{\prime}(0)=i k u_{\mp}(0) \mp i a m u_{ \pm}(0) .
\end{aligned}
$$

A measure of the strength of the coupling inflaton - fermions is given by the parameter

$q \equiv g^{2} \phi^{2}(0) / 4 m_{\phi}^{2}$,

where $\phi(0) \simeq 0.28 M_{p}$ is the value of the inflaton at the end of the inflation and $m_{\phi} \simeq 10^{13} \mathrm{GeV}$ is the inflaton mass.

In Fig.11 the results of a numerical integration of the equations given above are reported. For a wide range of $m_{X}$ and $q$ the value of the ratio $\rho_{X} / \rho$ is shown, where $\rho_{X}=m_{X} \cdot n_{X}$ is the energy density transferred to the fermions and $\rho$ is the initial energy density stored in the inflaton field.

One can notice the presence of the cut-off $m_{\max } \sim q^{1 / 2} / 2$ in the fermionic mass. For $m_{X}$ higher than this value the production is substantially zero, while for lower values it increases linearly with $q$ and is independent from $m_{X}$. This last statement seems not to be completely confirmed by Fig.11, where the value $\rho_{X}$ increases

\footnotetext{
${ }^{4}$ Note that with these values $n(0)=0$.
}

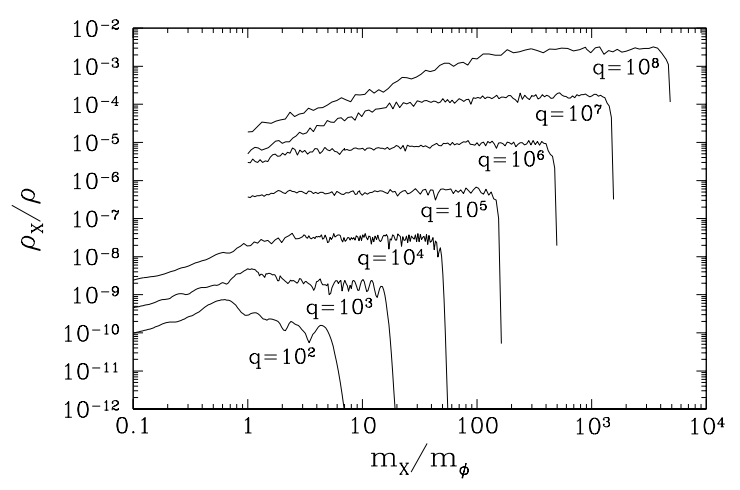

Figure 1. Ratio between the energy density of the produced fermions and of the inflaton field, for many values of the fermion mass and of $q$.

slowly with $m_{X}$. However, as the analytical estimate below will confirm, the scaling stated here is exact, while the slope that one sees in Fig.1 for high $q$ and small $m_{X}$ is a numerical artifact. The problem is that - due to limited computational resources $-\rho_{X}$ reported in Fig.11 is evaluated after 20 oscillations of the inflaton field. However, the number of oscillations of $\phi$ that one needs for $\rho_{X}$ to saturate increases with $q$, and a slope in $\rho_{X}$ appears for those values for which the saturation has not yet taken place.

To go on, it is important to understand the behavior of $n_{k}$, that is the number density of fermions produced with a given momentum $k$. All these spectra exhibit the qualitative behavior that is reported in Fig.2 for $m_{X}=100 m_{\phi}$ and for two values of $q$.

Also here one can find a threshold $k_{\max }$ in the production. For $k<k_{\max }, n_{k}$ approximately saturates to 1 (filling the whole allowed Fermi sphere), while for momenta bigger than $k_{\max }$ the production does not occur. Thus one can estimate [see Eq. (6)]

$\rho_{X}=m_{X} \cdot n_{X} \propto m_{X} \cdot k_{\max }^{3}$.

As it is shown in Fig.2, $k_{\max }$ increases with $q$. More precisely, the analytical estimate that will be given below indicates $k_{\max } \propto\left(q / m_{X}\right)^{1 / 3}$. 


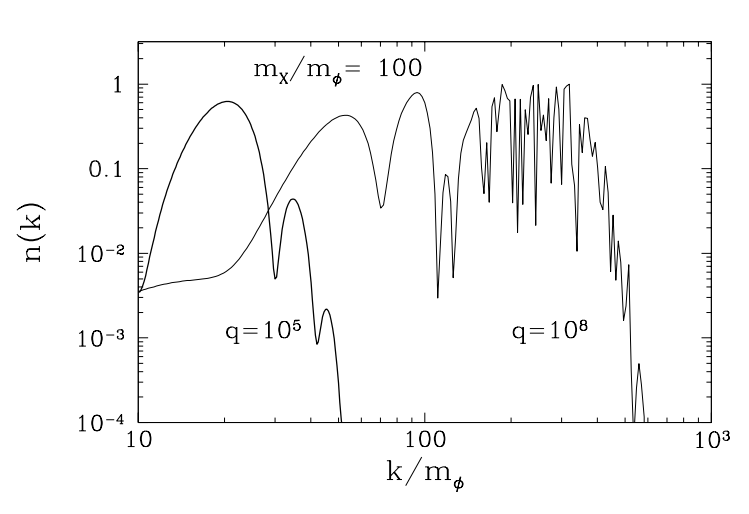

Figure 2. Spectrum in momenta of the produced particles for a given fermion mass and two different values of $q$.

From this value and from Eq. (10) one immediately gets the scaling $\rho_{X} \propto q$ stated above.

To understand the scaling of $k_{\max }$, one can differentiate Eq. (6):

$\frac{d n_{k}}{d \eta}=-\frac{1}{\omega^{2}} \frac{d \omega}{d \eta} \frac{d^{2}\left|u_{+}\right|^{2} / d \eta^{2}}{8(m a)}$.

This expression suggests that $n_{k}$ changes significantly when the total mass $m$ approaches zero and when the variation of the frequency $\omega$ is nonnegligible. Indeed, our numerical results indicate that the production of fermions occurs for very short intervals about the zeros of $m$. The estimate that is here presented consists in neglecting the last factor in Eq. (11) and, as it is customary done for preheating of bosons, in assuming that the production occurs only for $\left|\omega^{\prime} / \omega^{2}\right|>1$, that is in a regime of non adiabatic change of the frequency .

In terms of the number $N$ of oscillations of the inflaton field, the non adiabaticity condition

\footnotetext{
${ }^{5}$ The results that will be presented here can be obtained (in a more lengthy way) also without this assumptions and hence do not depend on the approximations made here.
}

rewrites

$$
\frac{k^{2}}{a^{2}}<\left(\frac{m}{2 \pi} \frac{d m}{d N}\right)^{2 / 3}-m^{2},
$$

where masses and momenta are given in units of the inflaton mass.

The cut-off in the production for $m_{X}>m_{\max }$ can be now understood by rewriting Eq. (4) in terms of $N$ :

$m=m_{X}+2 q^{1 / 2} \frac{\phi}{\phi(0)} \approx m_{X}+\frac{q^{1 / 2}}{\pi N} \cos 2 \pi N$

At large $m_{X}$ the first zero of $m$ can be potentially reached at $N=1 / 2$. However, for $m_{X}$ greater than $2 q^{1 / 2} / \pi$, the total mass $m$ never vanishes and Eq. (12) is never satisfied 6 .

For $m_{X}<m_{\max }$, the total mass vanishes at the points $N_{0}$ given by $\left(N_{\max }\right.$ is the greatest $\left.N_{0}\right)$

$N_{0}=-\frac{q^{1 / 2}}{\pi m_{X}} \cos 2 \pi N_{0}, \quad N_{\max } \simeq \frac{q^{1 / 2}}{\pi m_{X}}$.

To find $k_{\max }$, one has to maximize r.h.s. of Eq. (12). The maximum occurs when the two terms in r.h.s. are of the same order. Thus, a good estimate for $k_{\max }$ can be achieved by solving

$$
\left(\frac{m m^{\prime}}{2 \pi}\right)^{2 / 3} \simeq m^{2} \simeq\left(\frac{k}{a}\right)^{2}
$$

expanding $m$ and $m^{\prime}$ for $N$ close to $N_{0}$. Doing so, one gets

$k \simeq a m \sim\left|\cos 2 \pi N_{0}\right|^{1 / 3} q^{1 / 6} N_{0}^{1 / 3}$.

Since $k$ grows with $N_{0}, k_{\max }$ has to be evaluated in $N_{\max }$. Thus the particles with the highest momentum are generated in the last zero $N_{\max }$ of $m$. This gives the stated result

$k_{\max } \propto\left(\frac{q}{M}\right)^{1 / 3}$.

\footnotetext{
${ }^{6}$ While numerical coefficient based on the second approximate equality in the r.h.s of Eq. (13) should not being taken seriously, numerical integration shows that $2 \phi / \phi(0) \approx-0.5$ in the point of first minimum of $\phi$, which gives the cut-off in the proper place, $m_{X} \approx q^{1 / 2} / 2$.
} 


\section{Baryogenesis}

The previous section shows that preheating can produce fermions with the energy density

$$
\left(\frac{\rho_{X}}{\rho_{\phi}}\right) \sim 10^{-11} q \text { up to } M_{X}=\frac{q^{1 / 2}}{2} m_{\phi} .
$$

This result can be useful for leptogenesis if one identifies the $X$ particles with right-handed neutrinos $N$ and if these heavy neutrinos decay in a $C P$ violating manner into left handed neutrinos plus higgs particles.

Eq. (18) is obtained with the tacitly assumption that the produced fermions are stable. However this estimate does not apply if the right handed neutrinos have a decay lifetime larger than the typical time-scale of the inflaton oscillation $m_{\phi}^{-1}$.

Another requirement that must be put is that the right handed neutrinos do not annihilate into inflaton quanta more rapidly than they decay in left handed neutrinos, otherwise the produced lepton asymmetry would be negligible.

Both the above requirements can be typically satisfied Ø for masses $M_{N} \leq 10^{15} \mathrm{GeV}$ and for couplings $q \leq 10^{10}$.

Once the right handed neutrinos decay and once the lepton asymmetry produced by the decay is converted in baryon asymmetry by sphaleron transitions, Eq. (18) gives [1]

$$
\begin{gathered}
B \equiv \frac{n_{B}}{s}= \\
3 \cdot 10^{-7} \varepsilon\left(\frac{T_{r h}}{10^{10} \mathrm{GeV}}\right)\left(\frac{10^{15} \mathrm{GeV}}{M_{N}}\right)\left(\frac{q}{10^{10}}\right),
\end{gathered}
$$

where $T_{r h}$ is the reheating temperature and $\varepsilon$ is the amount of $C P$ violation in the decay of the heavy neutrinos 8 .

Eq (19) reproduces for very natural values of the parameters the observed baryon asymmetry

\footnotetext{
${ }^{7}$ One must also ask that lepton number violating scatterings do not deplete the lepton asymmetry generated bye the decay of the right handed neutrinos. See [1] for details. ${ }^{8} \mathrm{Eq}(19)$ holds if the energy density of the right handed neutrinos never dominates over the thermal one. However, if the neutrinos have a very late decay they can eventually dominate over the thermal bath, since their energy does not redshift with the expansion of the Universe. If it is the case, the decay of the neutrinos gives both the baryon number and the entropy of the thermal bath, and
}

of the Universe. This result leads thus to conclude that baryogenesis via leptogenesis is a viable option also if one considers right-handed neutrinos with mass higher than the inflaton one.

\section{Acknowledgements}

I would like to thank G.F. Giudice, A. Riotto, and I. Tkachev, with whom the results presented in this talk were obtained. I'm also grateful to the organizators of the Conference for the very friendly and stimulating atmosphere. This work is partially supported by the EEC TMR network "Beyond the Standard Model", contract no. FMRX-CT96-0090.

\section{REFERENCES}

1. G.F. Giudice, M. Peloso, A. Riotto, and I. Tkachev, hep-ph/9905242.

2. A. Riotto and M. Trodden, hep-ph/9901362, to appear in Annual Review of Nuclear and Particle Science.

3. M. Gell-Mann, P. Ramond and R. Slanski, in Supergravity, ed. P. Van Nieuwenhuizen and D.Z. Freedman, North Holland (1979).

4. A.D. Dolgov and A.D. Linde, Phys. Lett. B116, 329 (1982).

5. L.F. Abbott, E. Farhi, and M. Wise, Phys. Lett. B117, 29 (1982).

6. J. Ellis, J. Kim, and D.V. Nanopoulos, Phys. Lett. B145, 181 (1984).

7. L.A. Kofman, A.D. Linde, and A.A. Starobinsky, Phys. Rev. Lett. 73, 3195 (1994).

8. S.Yu. Khlebnikov and I. Tkachev, Phys. Rev. Lett. 77, 219 (1996), Phys. Lett. B390, 80 (1997), Phys. Rev. Lett. 79, 1607 (1997), Phys. Rev. D56, 653 (1997).

9. E.W. Kolb, A.D. Linde and A. Riotto, Phys. Rev. Lett. 77, 4290 (1996).

10. E.W. Kolb, A. Riotto and I. Tkachev, Phys. Lett. B423, 348 (1998).

the resulting baryon asymmetry is given by

$B=8 \cdot 10^{-3}\left(\frac{m}{10^{-6} \mathrm{eV}}\right)^{1 / 2} \varepsilon$,

where $m$ is the mass of the light neutrinos. 